\title{
On the Vacuum State in Quantum Field Theory. II
}

\author{
By
}

\section{H. J. Bor ChERS}

\author{
Institut des Hautes Etudes Scientifiqes \\ Bures-sur-Yvette (S. \& O.) France
}

\begin{abstract}
We want to construct, for every local irreducible quantum field theory which fulfils the spectrum condition, a new theory with the properties:

1) It is physically equivalent to the given theory (in the sense of HAAG and KASTLER).

2) The representation space contains a vacuum state.

3) The new theory satisfies the spectrum condition.

4) For every bounded region $\mathcal{O}$ the two representations of the algebra $\mathfrak{A}(\mathcal{O})$ are unitarily equivalent.

5) The new theory is uniquely characterized by the properties 1)-4).
\end{abstract}

\section{Introduction}

In the usual treatment of quantum field theory one assumes the existence of a vacuum state, i.e. of a state which is invariant under all Translations. In an earlier paper [1] this postulate was discussed. We showed the possibility of associating to every field theory another theory in which the Hilbert space also contains a vacuum state. However, we proved only the existence of a translation-invariant positive functional on the algebra generated by all local rings. We did not know at that time what properties the theory described by this functional would have. It is the aim of this note to fill this gap. The results we get are those expected intuitively from the "particle behind the moon" argument. This confirms that the vacuum assumption is only a postulate which one can add for convenience.

The frame for our investigations will be the theory of local rings of observables which has been developped in recent years by ARAKI, HaAG and KASTLER [2]. For our purpose the existence of a unitary representation of the translation group fulfilling the spectrum condition is very essential. This implies that we cannot adopt the purely algebraic view of the theory of local ring systems. But even if the problem had a purely algebraic aspect the author would not omit the spectrum condition, for the following reasons:

From the well studied equal-time commutation relation it is known that the number of inequivalent faithful representations of a free Bosefield is at least that of the continuum. SEgaL [3] showed that there 
even exists a one-parametric family of inequivalent representations having translation-invariant states. But in only one representation is the energy-operator non-negative. In analogy to this we have to expect for an arbitrary local ring system also a non-countable number of inequivalent representations, and also that in most of these representations the energy will not be positive.

On the other hand one would like to link the notion of "physical equivalence" to superselection-rules. Since experimentalists have associated superselection-rules to quantum numbers like charge, nucleon number etc., one would prefer a notion of physical equivalence which leads to only a countable number of representations. This seems to indicate that not all inequivalent, equally faithful representations fulfil every assumption made implicitly while doing experiments.

Since we do not know exactly which information is missing, we only can hope that the spectrum condition gives the right selection-principle. In Segal's example this was just the condition which was needed to get a unique representation of the given ring system in a Hilbert space with vacuum.

We will not achieve an equally strong result. But we will show that the spectrum condition leads in a natural manner to classes of representations which are locally unitarily equivalent. This means, for every bounded open region the equivalence can be established by a unitary mapping. This unitary operator, however, depends on the region and there exists no unitary operator which does the mapping for all open regions simultaneously. Within such a class we get again the result that the theory with vacuum and positive energy is unique.

Another aspect of our result comes from the usual field theory in terms of boson and fermion field operators. In such a theory the superselection-rules are given in a natural manner in terms of the "charges *." In every charge sector we have a local ring system which is generated by the neutral boson field and by the neutral bilinear expressions in the charged fields. The charged fields $\psi(x)$, however, give rise to operators which map one charge sector into another. If we consider a bounded region $\mathcal{O}$ and the associated ring $\mathfrak{R}(\mathcal{O})$ then we can find a test function $f(x)$ whose support is spacelike with respect to $\mathcal{O}$. The operator $\psi(f)$ then maps the ring $\mathfrak{R}_{1}(\mathcal{O})$ in the one charge sector onto the corresponding $\mathfrak{R}_{2}(\mathcal{O})$ in another charge sector. If we make a polar decomposition of $\psi(f)$ we get a unitary operator which does this mapping. As we change $\mathcal{O}$ we have to change the test function $f(x)$. We see from this that we get local unitary equivalence between the different charge sectors but not a global one.

* We mean not only quantities like the electric and nucleonic charge, but also the distinction between integral and halfintegral spin. 


\section{Assumptions}

We assume we have assigned to every bounded open region $\mathcal{O}$ in the Minkowski space a ring $\mathfrak{A}(\mathcal{O})$ of bounded operators in a separable Hilbert space $\mathfrak{S}$. Moreover, we assume there exists in $\mathfrak{S}$ a unitary representation $U(x)$ of the translation group. $U(x)$ and $\mathfrak{A}(\mathcal{O})$ shall have the properties:

1) The spectrum of $U(x)$ is contained in the closed forward lightcone $\overline{\mathscr{V}}+$.

2) $\mathfrak{A}(\mathcal{O})$ and $\mathfrak{A}(\mathcal{O}+x)$ are related by the equation

$$
\mathfrak{A}(\mathcal{O}+x)=U(x) \mathfrak{A}(\mathcal{O}) U^{-1}(x) \text {. }
$$

3) $\mathfrak{A}(\mathcal{O})$ is closed in the norm topology and is generated by its selfadjoint elements. This means $\mathfrak{A}(\mathcal{O})$ is a $C^{*}$-algebra.

4) If two regions $\mathcal{O}_{1}$ and $\mathcal{O}_{2}$ are spacelike to one another, then the corresponding rings $\mathfrak{A}\left(\mathcal{O}_{1}\right)$ and $\mathfrak{A}\left(\mathcal{O}_{2}\right)$ commute.

5) If $\mathcal{O}_{1} \subset \mathcal{O}_{2}$, then $\mathfrak{A}\left(\mathscr{O}_{1}\right) \subset \mathfrak{A}\left(\mathcal{O}_{2}\right)$.

6) If $\left\{\mathcal{O}_{n}\right\}$ is any covering of the Minkowski space by bounded open regions, then the weak closure of the algebra generated by the family $\left\{\mathfrak{A}\left(\mathcal{O}_{n}\right)\right\}$ is independent of the covering.

We do not want to make any more assumptions about the structure of the global v. Naumenn algebra $\mathfrak{R}(\mathscr{M})$. From these assumptions it follows already that the center of $\mathfrak{R}(\mathscr{M})$ (which we denote by $\mathfrak{\complement}(\mathscr{M})$ ) is pointwise invariant under the action of the translation group

$$
U(x) A U^{-1}(x)=A \quad \text { if } \quad A \in \mathcal{E}(\mathscr{M}) .
$$

This result is due to Araki [4].

We refer to condition 6 ) as the weak additivity property. This is the weakest known assumption to get the Reeh-Schlieder theorem. [5]. For v. Naumenn algebras we will always use the letter $\mathfrak{R}$. In particular $\mathfrak{R}(\mathcal{O})$ is the weak closure of $\mathfrak{A}(\mathcal{O})$ and $\mathfrak{R}(\mathscr{M})$ is the weak closure of $\mathfrak{A}(\mathscr{M})$. With this notation condition 6 ) can be rephrased as follows:

$6^{\prime}$ ) If $\left\{\mathcal{O}_{n}\right\}$ is any covering of the Minkowski space by bounded open sets, then the $v$. Neumann algebra generated by the family $\left\{\mathfrak{R}\left(\mathcal{O}_{n}\right)\right\}$ is equal to $\mathfrak{R}(\mathscr{M})$.

We have assumed that $\mathfrak{A}(\mathcal{O})$ are $C^{*}$-algebras. Since every $\nabla$. Neumann algebra is also a $C^{*}$-algebra it is allowed to think that $\mathfrak{A}(\mathcal{O})$ and $\mathfrak{R}(\mathcal{O})$ are the same. However $\mathfrak{A}(\mathscr{M})$ and $\mathfrak{R}(\mathscr{M})$ will never be the same.

With $\mathfrak{R}^{\prime}$ we denote the commutant of $\mathfrak{R}$.

\section{Definition of the vacuum functional}

The definition of the vacuum functional is based on the following:

Theorem 1. Assume we have a theory fulfilling 1) through 6). Let $a$ be a spacelike vector. The there exists a sequence $\left\{\lambda_{n}\right\}, \lambda_{n} \rightarrow \infty$ such 
that $U\left(\lambda_{n} a\right) B U^{-1}\left(\lambda_{n} a\right)$ converges weakly for every $B \in \mathfrak{A}(\mathscr{M})$. The limit elements

$$
L(B)=\text { weak } \lim _{n \rightarrow \infty} U\left(\lambda_{n} a\right) B U^{-1}\left(\lambda_{n} a\right)
$$

are contained in the center of $\mathfrak{R}(\mathscr{M})$. They are linear functions on the algebra and they are non-negative for non-negative elements. Moreover $L(B)=L\left(U(x) B U^{-1}(x)\right)$ for all $x$.

An immediate consequence of this theorem is the following

Corollary 2. We add to the conditions of Theorem 1 the assumption that $\mathfrak{R}(\mathscr{M})$ is a factor. Then, for every $B \in \mathfrak{A}(\mathscr{M}), L(B)$ is of the form $\omega(B) 1$ where $\omega$ is a translation-invariant positive linear functional on the algebra $\mathfrak{A}(\mathscr{M})$.

Proof of the theorem:

Let us denote by $\mathfrak{E}(\mathscr{M})$ the center of $\mathfrak{R}(\mathscr{M})$. $\mathfrak{\complement}(\mathscr{M})$ is an Abelian algebra. If $\Psi$ is a separating vector for $\mathfrak{S}(\mathscr{M})$ then $U(x) \Psi$ is again a separating vector for $\mathfrak{E}(\mathscr{M})$ since every element of $\mathfrak{\mathfrak { C }}(\mathscr{M})$ commutes with $U(x)$. Since $\mathcal{G}$ is a separable Hilbert space we can pick a vector $\Psi$ which is separating for $\mathfrak{C}(\mathscr{M})^{\star}$.

We define a larger algebra $\hat{\mathfrak{A}}(\mathscr{M})$, which is the $C^{*}$-algebra generated by $\mathfrak{A}(\mathscr{M})$ and the center $\mathfrak{E}(\mathscr{M})$. Consider now the set of functionals $(U(-\lambda a) \Psi, B U(-\lambda a) \Psi)$ on $\hat{\mathfrak{A}}(\mathscr{M})$ for a fixed spacelike vector. This is, of course, a bounded set of positive functionals. It is known that the positive functionals of norm 1 form a weakly compact set in the topology induced by the self-adjoint elements of $\hat{\mathfrak{A}}(\mathscr{M})$ (see [6], § 3, subsection 7, III). We therefore can pick a subsequence $\left\{\lambda_{n}\right\}, \lambda_{n} \rightarrow \infty$, so that

$$
\lim _{n \rightarrow \infty}\left(U\left(-\lambda_{n} a\right) \Psi, B U\left(-\lambda_{n} a\right) \Psi\right)=\omega(B)
$$

exists for all $B$ from $\hat{\mathfrak{A}}(\mathscr{M})$. We have clearly $\omega\left(B^{*} B\right) \geqq 0$.

This means $\omega$ is a non-negative functional.

We now want to show that $U\left(\lambda_{n} a\right) B U\left(-\lambda_{n} a\right) \Psi$ converges. We do this in several steps.

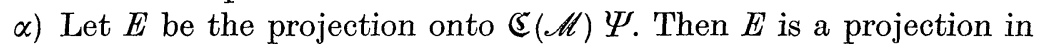
$\mathfrak{C}(\mathscr{M})^{\prime}$. We now want to show that for $\Phi$ with $E \Phi=0$ we get

$$
\lim _{n \rightarrow \infty}\left(\Phi, U\left(\lambda_{n} a\right) B U\left(-\lambda_{n} a\right) \Psi\right)=0 .
$$

To prove this we give an $\varepsilon>0$. Since $\hat{\mathfrak{A}}(\mathscr{M})$ is generated by all $\mathfrak{A}(\mathcal{O})$ and the center, we can find an $\mathcal{O}$, and an operator $B_{1}$ in the algebra $\hat{\mathfrak{A}}(\mathcal{O})$ generated by $\mathfrak{A}(\mathcal{O})$ and the center, such that $\left\|B-B_{1}\right\| \leqq \frac{\varepsilon}{3} \times$ $\times\|\Psi\|^{-1}\|\Phi\|^{-1}$.

* At this point we need the assumption that $\mathscr{H}$ is separable. This assumption is not essential, but the proofs are much more elaborate without it. 
On the other hand $\mathfrak{S}\left(\mathscr{M}^{\prime}\right)$ is generated by all rings $\mathfrak{R}(\mathcal{O})$ and $\mathfrak{R}(\mathscr{M})^{\prime}$. This means we can find an $\mathcal{O}_{2}$ and an operator $E_{1}$ in the ring $\mathfrak{R}\left\{\mathfrak{R}\left(\mathcal{O}_{2}\right)\right.$, $\left.\mathfrak{R}(\mathscr{M})^{\prime}\right\}$ generated by $\mathfrak{R}\left(\mathcal{O}_{2}\right)$ and $\mathfrak{R}(\mathscr{M})^{\prime}$, such that

and

$$
\left\|E_{1} \Psi-\Psi\right\| \leqq \frac{\varepsilon}{3}\|\Phi\|^{-1}\left\|B_{1}\right\|^{-1}
$$

$$
\left\|E_{1}^{*} \Phi\right\| \leqq \frac{\varepsilon}{3}\|\Psi\|^{-1}\left\|B_{1}\right\|^{-1}
$$

We choose now $N$ in such a way that $\mathcal{O}_{1}+\lambda_{n} a$ and $\mathcal{O}_{2}$ are spacelike to one another for $n>N$. Then we have

$$
\begin{aligned}
& \left\|\left(\Phi, U\left(\lambda_{n} a\right) B U\left(-\lambda_{n} a\right) \Psi\right)\right\| \leqq \\
& \quad \leqq\left\|\left(\Phi, U\left(\lambda_{n} a\right) B_{1} U\left(-\lambda_{n} a\right) \Psi\right)\right\|+\frac{\varepsilon}{3} \leqq \\
& \quad \leqq\left\|\left(\Phi, U\left(\lambda_{n} a\right) B_{1} U\left(-\lambda_{n} a\right) E_{1} \Psi\right)\right\|+\frac{2}{3} \varepsilon \\
& \quad=\left\|\left(E_{1}^{*}, \Phi, U\left(\lambda_{n} a\right) B_{1} U\left(-\lambda_{n} a\right) \Psi\right)\right\|+\frac{2}{3} \varepsilon \leqq \varepsilon \quad \text { if } \quad n>N .
\end{aligned}
$$

ק) Let now $E \Phi=\Phi$.

Then by definition of $E$ there exists an element $A \in \mathbb{E}(\mathscr{M})$ such that

This implies

$$
\|A \Psi-\Phi\| \leqq \frac{\varepsilon}{3}\|B\|^{-1}\|\Psi\|^{-1}
$$

$$
\begin{aligned}
\|(\Phi, U & \left.\left(\lambda_{n} a\right) B U\left(-\lambda_{n} a\right) \Psi\right)-\left(\Phi, U\left(\lambda_{p} a\right) B U\left(-\lambda_{p} a\right) \Psi\right) \| \leqq \\
& \leqq\left\|\left(A \Psi,\left(\lambda_{n} a\right) B U\left(-\lambda_{n} a\right) \Psi\right)-\left(A \Psi, U\left(\lambda_{p} a\right) B U\left(-\lambda_{p} a\right) \Psi\right)\right\|+\frac{2}{3} \varepsilon \\
& =\left\|\left(\Psi, U\left(\lambda_{n} a\right) A^{*} B U\left(-\lambda_{n} a\right) \Psi\right)-\left(\Psi, U\left(\lambda_{p} a\right) A^{*} B U\left(-\lambda_{p} a\right) \Psi\right)\right\|+ \\
& +\frac{2}{3} \varepsilon \leqq \varepsilon \quad \text { for } \quad n, p>N .
\end{aligned}
$$

$\alpha)$ and $\beta$ ) together imply $U\left(\lambda_{n} a\right) B U\left(-\lambda_{n} a\right) \Psi$ converges weakly.

We now want to show that $U\left(\lambda_{n} a\right) B U\left(-\lambda_{n} a\right)$ is a weakly convergent sequence of operators.

Since this is a bounded sequence it is sufficient to show the convergence of the sequence on a dense set. Since $\Psi$ has been chosen to be a separating vector for $\mathfrak{C}(\mathscr{M})$ we know that $\mathfrak{S}(\mathscr{M})^{\prime} \Psi$ is a dense set in the Hilbert space. $\mathfrak{S}(\mathscr{M})^{\prime}$, on the other hand, was generated by an increasing sequence of rings $\mathfrak{R}\left\{\mathfrak{R}\left(\mathcal{O}_{n}\right), \mathfrak{R}(\mathscr{M})^{\prime}\right\}$. This means, it is sufficient to show the convergence on all vectors of the form $\mathfrak{R}\left\{\mathfrak{R}(\mathcal{O}), \mathfrak{R}\left(\mathscr{M}^{\prime}\right\}\right) \Psi$ for all $\mathcal{O}$.

Let now $A \in \mathfrak{R}\left\{\mathfrak{R}(\mathcal{O}), \mathfrak{R}(\mathscr{M})^{\prime}\right\}$ and $B \in \mathfrak{A}(\mathscr{M})$ and $\varepsilon>0$ and $\Phi \in \mathfrak{S}$. We choose $B_{1}$ from a certain $\mathfrak{A}\left(\mathcal{O}_{1}\right)$ such that

$$
\left\|B-B_{1}\right\|<\frac{\varepsilon}{3}\|\Phi\|^{-1}\|A \Psi\|^{-1} \text {. }
$$


Then we get for sufficiently large $n, p$

$$
\begin{aligned}
\mid(\Phi, U & \left.\left(\lambda_{n} a\right) B U\left(-\lambda_{n} a\right) A \Psi\right)-\left(\Phi, U\left(\lambda_{p} a\right) B U\left(-\lambda_{p} a\right) A \Psi\right) \mid \leqq \\
& \leqq\left|\left(\Phi, U\left(\lambda_{n} a\right) B_{1} U\left(-\lambda_{n} a\right) A \Psi\right)-\left(\Phi, U\left(\lambda_{p} a\right) B_{1} U\left(-\lambda_{p} a\right) A \Psi\right)\right|+ \\
& +\frac{2}{3} \varepsilon=\mid\left(A^{*} \Phi, U\left(\lambda_{n} a\right) B_{1} U\left(-\lambda_{n} a\right) \Psi\right)- \\
& -\left(A^{*} \Phi, U\left(\lambda_{p} a\right) B_{1} U\left(-\lambda_{p} a\right) \Psi\right) \mid+\frac{2}{3} \varepsilon \leqq \varepsilon
\end{aligned}
$$

for $n, p>N$ sufficiently large. This implies the weak limit $L(B)$ exists. If $B$ is a non negative self-adjoint operator, then $L(B)$ is as limit of non-negative operators again self-adjoint and non-negative. And from the definition of $L(B)$ it follows that $L(B)$ is linear in $B$. We also see that $\|L(B)\| \leqq\|B\|$. This means $L$ is a continuous linear map of $\mathscr{A}(\mathscr{M})$ into the bounded operators. To show that $L(\mathfrak{A}(\mathscr{M}))$ is contained in the center of $\mathfrak{R}(\mathscr{M})$ it is sufficient to show this for a dense set of $\mathfrak{A}(\mathscr{M})$. If $B \in \mathfrak{A}(\mathcal{O})$, then $L(B)$ clearly commutes with $\mathfrak{R}(\mathscr{M})^{\prime}$ and all $\mathfrak{R}(\mathcal{O})$. Since these rings generate $\mathfrak{C}(\mathscr{M})^{\prime}$ we have $L(B) \in \mathfrak{C}(\mathscr{M})$ and hence $L(\mathscr{H}(\mathscr{M})) \subset \mathfrak{C}(\mathscr{M})$.

That $L$ is an invariant map follows from the fact that the translation group is Abelian and the center contains only invariant elements. We have

$$
\begin{aligned}
L(U(x) B U(-x)) & =\text { weak } \lim U\left(\lambda_{n} a\right) U(x) B U(-x) U\left(-\lambda_{n} a\right) \\
& =U(x)\left(\text { weak } \lim U\left(\lambda_{n} a\right) B U\left(-\lambda_{n} a\right)\right) U(-x) \\
& =U(x) L(B) U(-x)=L(B) .
\end{aligned}
$$

This proves the theorem.

At this stage we are unfortunately not able to show that this limit $L(B)$ exists independent of the choice of the spacelike vector $a$ and independent of the selected sequence $\left\{\lambda_{n}\right\}$. This implies in particular, if we had started with a theory covariant under the inhomogeneous Lorentz group we would have to drop this additional invariance property. This gap can be filled, but we have to wait until the very end.

\section{Some facts about local ring systems}

This section is devoted to problems which can be treated by analytic methods made available by the spectrum condition. We need the following notations:

Definition 3. Let $E_{\Delta}$ be the spectral projections of the translation group, and $\Psi \in \mathfrak{G}$. We define support $\Psi$ as the smallest closed subset $\Delta$ of the spectrum of the translation group such that $E_{4} \Psi=\Psi$.

Definition 4. A vector $\Psi \in \mathfrak{G}$ is called an analytic vector for the energy-operator $P_{0}$ if $\Psi$ is in the domain of $P_{0}^{n} n=1,2, \ldots$ and the 
series

$$
\sum\left\|P_{0}^{n} \Psi\right\| \frac{z^{n}}{n !}
$$

has a non-zero radius of convergence.

Lemma 5. (REEH-SCHLIEDER [5]).

Let $\Psi \in \mathfrak{S}$ have the two properties

1) $\Psi$ is an analytic vector for the energy,

2) $\Psi$ is separating for $\mathfrak{C}(\mathscr{M})$.

Then $\Psi$ is cyclic for $\mathfrak{R}\left\{\mathfrak{R}(\mathcal{O}), \mathfrak{R}(\mathscr{M})^{\prime}\right\}$ and separating for $\mathfrak{R}(\mathcal{O})$, and this for every bounded open region $\mathcal{O}$.

Proof. Since $\Psi$ is an analytic vector for the energy this means

$$
\sum\left\|P_{0}^{n} \Psi\right\| \frac{z^{n}}{n !} \quad \text { converges for } \quad|z|<a, \quad a>0
$$

we see that $U(x) \Psi$ is an analytic function with values in a Hilbert space holomorphic in the tube $\operatorname{Im} x \in-a e_{0}+\mathscr{V}^{+}$, where $e_{0}$ is the unit vector in the time direction.

We pick now a region $\mathcal{O}_{1} \subset \mathcal{O}$ such that $\mathcal{O}_{1}+x<\mathcal{O}$ for all $x$ in a small neighbourhood $\mathscr{N}$ of the origin. Assume $\Phi$ is in the orthogonal complement of $\mathfrak{R}\left\{\mathfrak{R}(\mathcal{O}), \mathfrak{R}(\mathscr{M})^{\prime}\right\} \Psi$. We want to show that $\Phi$ is zero.

Let $B_{1}, B_{2}, \ldots, B_{n}$ be elements from $\mathfrak{R}\left\{\mathfrak{R}\left(\mathcal{O}_{1}\right), \mathfrak{R}^{\prime}(\mathscr{M})\right\}$ then

$$
\begin{gathered}
\left(\Phi, U\left(x_{1}\right) B_{1} U\left(x_{2}-x_{1}\right) B_{2} U\left(x_{3}-x_{2}\right) \cdots U\left(x_{n}-x_{n-1}\right) B_{n} U\left(-x_{n}\right) \Psi\right)=0 \\
\text { for } x_{i} \in \mathscr{N} .
\end{gathered}
$$

On the other hand this function is the boundary value of an analytic function holomorphic in the tube

$$
\begin{gathered}
\operatorname{Im} x_{1} \in \mathscr{V}^{+} \cap \operatorname{Im}\left(x_{2}-x_{1}\right) \in \mathscr{V}^{+} \cap \cdots \\
\cdots \cap \operatorname{Im}\left(x_{n}-x_{n-1}\right) \in \mathscr{V}^{+} \cap \operatorname{Im}\left(x_{n}\right) \in a e_{0}+\mathscr{V}-.
\end{gathered}
$$

Since $a$ is a positive constant this region is not empty. It contains e.g. a neighbourhood of the point: $\vec{x}_{j}=0$

$$
0<\operatorname{Im} x_{1}^{0}<\operatorname{Im} x_{2}^{0}<\cdots<\operatorname{Im} x_{n}^{0}<a .
$$

A standard argument on analytic functions (see [7]) implies then that the above function vanishes for all values of $x$. By means of assumption 6 ) it follows that $\left(\Phi, \mathfrak{R}\left\{\mathfrak{R}(\mathscr{M}), \mathfrak{R}(\mathscr{M})^{\prime}\right\} \Psi\right)=0$. Since we have assumed that $\Psi$ is a cyclic vector for $\mathfrak{R}\left\{\mathfrak{R}(\mathscr{M}), \mathfrak{R}(\mathscr{M})^{\prime}\right\}$, we find $\Phi=0$. This implies $\Psi$ is cyclic for $\mathfrak{R}\left\{\mathfrak{R}(\mathcal{O}), \mathfrak{R}(\mathscr{M})^{\prime}\right\}$. Since $\mathcal{O}$ is a bounded region, $\Psi$ is also a cyclic vector for $\mathfrak{R}(\mathcal{O})^{\prime}$, the commutant of $\mathfrak{R}(\mathcal{O})$. But this implies $\Psi$ is separating for $\mathfrak{R}(\mathcal{O})$. This proves the lemma.

Theorem 6. Assume $\Psi_{1}, \ldots, \Psi_{n}$ are analytic vectors for the energy. Let $B_{1}, \ldots, B_{n} \in \mathfrak{R}(\mathcal{O})$ and assume $\sum_{1}^{n} B_{j} \Psi_{j}=0$. Then there exist $C_{i k} \in \mathfrak{E}(\mathscr{M}), i, k=1, \ldots, n$, such that $C_{i k}^{*}=C_{k i}$ and $\sum_{k} C_{i k} C_{k l}=C_{i \iota}$ 
and moreover

$$
\sum_{k} C_{i k} \Psi_{k}=\Psi_{i} \quad \text { and } \quad \sum_{j} B_{j} C_{j k}=0 .
$$

If in particular $\Psi_{k}$ is a separating vector for $\mathfrak{E}(\mathscr{M})$ then $C_{k k}$ is invertable.

Corollary 7. Let $\Psi \in \mathfrak{S}$ be an analytic vector for the energy and separating for $\mathfrak{E}(\mathscr{M})$.

If $B \in \mathfrak{R}(\mathcal{O})$ such that $B \Psi$ is an analytic vector for the energy then $B \in \mathfrak{S}(\mathscr{M})$.

We show first that the corollary follows from the theorem. We set $B \Psi=\Phi$. Then we have the relation $B C_{11}+\mathbf{1} C_{21}=0$. Since $\Psi$ was separating for $\mathfrak{C}(\mathscr{M})$ we see that $C_{11}^{-1}$ exists. This implies

$$
B=-C_{21} C_{11}^{-1} \in \mathfrak{C}(\mathscr{M}) \text {. }
$$

Proof of Theorem 6. Form the space $\hat{\mathfrak{H}}=n \otimes \mathfrak{F}$ of all systems $\left\{\Psi_{1} \ldots \Psi_{n}\right\}$ with the inner product

$$
\left(\left\{\Psi_{1} \ldots \Psi_{n}\right\},\left\{\Phi_{1} \ldots \Phi_{n}\right\}\right)=\sum_{j=1}^{n}\left(\Psi_{j} \Phi_{j}\right) .
$$

In $\hat{\mathfrak{Y}}$ we define a subspace $\mathfrak{M}$ by the two properties

1) $\left\{\Phi_{1} \ldots \Phi_{n}\right\} \in \mathfrak{M}$ implies $\sum B_{j} \Phi_{j}=0$

2) $\left\{\Phi_{1} \ldots \Phi_{n}\right\} \in \mathfrak{M}$ implies $\left\{A \Phi_{1} \ldots A \Phi_{n}\right\} \in \mathfrak{M}$ for all $A \in \mathfrak{C}(\mathscr{M})^{\prime}$. $\mathfrak{M}$ is obviously a closed subspace of $\hat{\mathfrak{y}}$.

We first show that the vector $\left\{\Psi_{1} \ldots \Psi_{n}\right\}$ mentioned in Theorem 6 is an element of $\mathfrak{M}$. To this end let us consider an open region $\mathcal{O}_{1}$ such that $\mathcal{O}_{1}+x$ is spacelike to $\mathcal{O}$ for all $x$ from an open neighbourhood $\mathscr{N}$ of the origin.

For $A_{1} \ldots A_{p} \in \mathfrak{R}\left\{\mathfrak{R}\left(\mathcal{O}_{1}\right), \mathfrak{R}(\mathscr{M})^{\prime}\right\}$ and $x_{1} \ldots x_{p} \in \mathscr{N}$ we have with the notation $A(x)=U(x) A U(-x)$

$$
\sum_{i=1}^{n} B_{i} A_{1}\left(x_{1}\right) \ldots A_{p}\left(x_{p}\right) \Psi_{i}=A_{1}\left(x_{1}\right) \ldots A_{p}\left(x_{p}\right) \sum_{i=1}^{n} B_{i} \Psi_{i}=0 \text { for } x_{i} \in \mathscr{N} .
$$

The vectors $\Psi_{i}$ are assumed to be analytic vectors for the energy with the radii of convergence $a_{i}$. Let $a$ be the minimum of the $a_{i}$. The vector

$$
\sum_{i=1}^{n} B_{i} A_{1}\left(x_{1}\right) \ldots A_{p}\left(x_{p}\right) \Psi_{i}
$$

is therefore the boundary value of an analytic function with values in a Hilbert space. The domain of holomorphy is the tube with basis

$$
\begin{gathered}
\operatorname{Im}\left(x_{1}\right) \in \mathscr{V}+\cap \operatorname{Im}\left(x_{2}-x_{1}\right) \in \mathscr{V}+\cap \cdots \\
\cdots \cap \operatorname{Im}\left(x_{p}-x_{p-1}\right) \in \mathscr{V}^{+} \cap \operatorname{Im} x_{p} \in a e_{0}+\mathscr{V}-.
\end{gathered}
$$

Vanishing of the function $(*)$ on an open set of the boundary implies that $(*)$ is identically zero. Condition 6 ) of Section II implies therefore

$$
\sum_{i=1}^{n} B_{i} A \Psi_{i}=0 \quad \text { for all } \quad A \in \mathfrak{S}(\mathscr{M})^{\prime} .
$$

This shows $\left\{\Psi_{1} \ldots \Psi_{n}\right\}$ is an element of $\mathfrak{M}$. 
Since $\mathfrak{M}$ is a closed linear manifold in $\hat{\mathfrak{j}}$ there exists a projection $P$ in $\mathscr{L}(\hat{\mathfrak{F}})$ such that

$$
\mathfrak{M}=P \hat{\mathfrak{Y}} .
$$

Now every bounded operator in $\hat{\mathfrak{Y}}$ can be written as an $n \times n$ matrix of bounded operators in $\mathfrak{S}$. So

$$
P=\left(C_{i k}\right) .
$$

Since $P$ is a projection we have the relations

$$
C_{i k}^{*}=C_{k i} \text { and } \sum_{k} C_{i k} C_{k l}=C_{i l} .
$$

Since $\left\{\Psi_{1} \ldots \Psi_{n}\right\}$ is an element of $\mathfrak{M}$ we have

$$
P\left\{\Psi_{1} \ldots \Psi_{n}\right\}=\left\{\Psi_{1} \ldots \Psi_{n}\right\} \quad \text { or } \quad \sum_{k} C_{i k} \Psi_{k}=\Psi_{i} .
$$

Now $\mathfrak{M}$ is invariant under the action of $\left(\mathfrak{S}(\mathscr{M})^{\prime} \times \mathbf{1}\right)$.

Therefore $P$ is an element from $\mathfrak{C}(\mathscr{M}) \otimes \mathscr{L}\left(\mathbb{C}_{n}\right)$ ([8], Chap. I, $\S 2$, Prop. 4). This means

Let now $\Phi \in \mathfrak{S}$; then

$$
C_{i k} \in \mathfrak{C}(\mathscr{M}) .
$$

$$
\left\{B_{1}^{*} \Phi \ldots B_{n}^{*} \Phi\right\} \in \hat{\mathfrak{Y}} \ominus \mathfrak{M}
$$

because for every $\left\{\Psi_{1} \ldots \Psi_{n}\right\} \in \mathfrak{M}$ we have

$$
\left(\left\{B_{1}^{*} \Phi \ldots B_{n}^{*} \Phi\right\},\left\{\Psi_{1} \ldots \Psi_{n}\right\}\right)=\sum_{j}\left(B_{j}^{*} \Phi, \Psi_{j}\right)=\sum_{j}\left(\Phi, B_{j} \Psi_{j}\right)=0
$$

This implies

$$
\left\{B_{1}^{*} \Phi \ldots B_{n}^{*} \Phi\right\} \in \hat{\mathfrak{G}} \odot \mathfrak{M} \quad \text { for all } \quad \Phi \in \mathfrak{F}
$$

or

$$
\sum_{k} C_{i k} B_{k}^{*} \Phi=0 \quad \text { for all } \quad \Phi \in \mathfrak{G} .
$$

This implies $\sum_{k} C_{i k} B_{k}^{*}=0$ and taking the adjoint of this equation we get

$$
\sum_{k} B_{k} C_{k i}=0 \text {. }
$$

To show the last statement we see that $\Psi_{k}$ cyclic for $\mathfrak{C}(\mathscr{M})^{\prime}$ implies $\left\{0 \ldots 0 \Phi_{k} \ldots 0\right\} \in \hat{\mathfrak{G}} \odot \mathfrak{M}$ if and only if $\Phi_{k}=0$. This means $\Psi_{k}$ is cyclic for $\mathfrak{C}(\mathscr{M})^{\prime}$ if and only if $C_{i k} \Phi_{l c}=0$ implies $\Phi_{k}=0$. Now assume $C_{k k}$ has a nullvector $\Phi$; then we have

$0=\left(\Phi, C_{k k} \Phi\right)=\left(\Phi, \sum_{i} C_{k i} C_{i k} \Phi\right)=\sum_{i}\left(C_{i k} \Phi, C_{i k} \Phi\right)=\sum_{i}\left\|C_{i k} \Phi\right\|^{2}=0$.

This means $C_{i k} \Phi_{k}=0$ implies $\Phi_{k}=0$ if and only if $C_{k k} \Phi_{k}=0$ implies $\Phi_{k}=\mathbf{0}$. 
Since $C_{k k}$ is self-adjoint this means $C_{k k}$ has an inverse $C_{k k}^{-1}$. This proves the theorem.

Theorem 8. Let $\mathcal{O}$ be a bounded open region and $\mathcal{O}_{1}$ a subregion such that $\mathcal{O}_{1}+x \subset \mathcal{O}$ for all $x$ from a certain open neighbourhood $\mathscr{N}$ of the origin. Denote by $\mathfrak{E}(\mathcal{O})$ the center of $\mathfrak{R}(\mathcal{O})$. Then we have $\mathfrak{S}(\mathcal{O}) \cap \mathfrak{E}\left(\mathcal{O}_{1}\right) \subset$ C $\mathfrak{S}(\mathscr{M})$.

Proof. Let $\Psi \in \mathfrak{S}$ have the two properties

1) $\Psi$ is separating for $\mathfrak{E}(\mathscr{M})$.

2) $\Psi$ is an analytic vector for the energy $P_{0} \star$.

Let $A \in \mathfrak{S}(\mathcal{O}) \cap \mathfrak{E}\left(\mathcal{O}_{1}\right)$ and consider the matrix element

$$
\left(\Psi, A^{*} U(x) A \Psi\right) \text {. }
$$

This function is the boundary value of an analytic function holomorphic in the forward tube. On the other hand consider the expression

$$
\left(\Psi, U(x) A U(-x) A^{*} U(x) \Psi\right) \text {. }
$$

Since $\Psi$ is an analytic vector for the energy with radius of convergence $a>0$ we see that (**) is the boundary value of an analytic function holomorphic in the tube with basis

$$
\operatorname{Im} x \in \mathscr{V}-\cap-a e_{0}+\mathscr{V}+.
$$

Since $A$ and $A^{*}$ are from $\mathfrak{S}(\mathcal{O}) \cap \mathfrak{E}\left(\mathcal{O}_{1}\right)$ these functions coincide for $x \subset \mathscr{N}$. From the edge-of-the-wedge theorem [9] it follows that the functions are analytic continuations of each other. This means (*) is holomorphic in a certain neighbourhood of the origin. In particular,

$$
\left(\Psi, A^{*} U(t, 0) A \Psi\right)
$$

is holomorphic for $|t|<a_{1}>0$.

This implies $\Sigma\left(\Psi, A^{*} P_{0}^{n} A \Psi\right) \frac{z^{n}}{n !}$ converges for $|z|<a_{1}$.

From this it follows (see e.g. [10]) that

$$
\sum\left\|P_{0}^{n} A \Psi\right\| \frac{z^{n}}{n !}
$$

converges for $|z|<\frac{a_{1}}{2}>0$.

This implies $A \Psi$ is again an analytic vector for the energy. Since $\Psi$ is separating for $\mathfrak{S}(\mathscr{M})$ we have by Corollary $7 A \in \mathfrak{C}(\mathscr{M})$. This proves the theorem.

\section{The spectrum condition for the new theory}

In this section we start with the additional assumption that $\Re(\mathscr{M})$ is a factor. This is exactly the condition of Corollary 2. The vacuum

* Since every element from the center commutes with the translations, we see that for every vector $\Psi$ separating for the center, $\exp \left(-P_{0}\right) \Psi$ is also separating. 
functional is then defined by

$$
\lim _{n \rightarrow \infty}\left(\Psi U\left(\lambda_{n} a\right) B U\left(-\lambda_{n} a\right) \Psi\right)=\omega(B)
$$

and this definition is independent of $\Psi$. To demonstrate that the new theory fulfils the spectrum condition, we have to show that for every vector $\widetilde{\Psi} \in \widetilde{\mathfrak{S}}$ the Fourier transform of the function $(\widetilde{\Psi}, \widetilde{U}(x) \widetilde{\Psi})$ has its support in the closed forward lightcone $\overline{\mathscr{V}}+$. From continuity it follows that it is sufficient to show this for a dense set of vectors. Since $\widetilde{\mathfrak{A}}(\mathscr{M})$ is the cyclic representation we have by the Reeh-Schlieder theorem that $\widetilde{\mathfrak{U}}(\mathcal{O}) \Omega(\Omega$ the cyclic vacuum state $)$ is a dense set of vectors in $\widetilde{\mathfrak{S}}$. So it is sufficient to show that for all $\tilde{A} \in \widetilde{\mathfrak{A}}(\mathcal{O})$ the expression $\left(\Omega, \widetilde{A}^{*} \widetilde{U}(x) \widetilde{A} \Omega\right)$ has a Fourier transform with support in $\overline{\mathscr{V}}+$. This, however, is equivalent to: The Fourier transform of

$$
\omega\left(A^{*} U(x) A U(-x)\right)
$$

has its support contained in the forward lightcone. In our case $\omega$ is given by the following formula

$\omega\left(A^{*} U(x) A U(-x)\right)=\lim _{n \rightarrow \infty}\left(U\left(-\lambda_{n} a\right) \Psi, A^{*} U(x) A U(-x) U\left(-\lambda_{n} a\right) \Psi\right)$

and this formula is independent of $\Psi$. So we choose a $\Psi$ which has compact support. In this case

$$
\left(U\left(-\lambda_{n} a\right) \Psi, A^{*} U(x) A U(-x) U\left(-\lambda_{n} a\right) \Psi\right)
$$

is the Fourier transform of a function with support contained in

independent of $\lambda_{n}$.

$$
-\operatorname{supp} . \Psi+\overline{\mathscr{V}}+
$$

Now with every $A$ in $\mathfrak{A}(\mathcal{O})$ we have also

$$
\int d^{4} x U(x) A U(-x) f(x) \in \mathfrak{A}(\mathscr{M}) \text { for } f(x) \in \mathscr{D}^{\star} .
$$

Now since $\mathfrak{A}(\mathscr{M})$ is norm closed we see that (***) holds for all test-functions in $\mathscr{S}$. This means in particular that equation $(*)$ holds in $\mathscr{S}^{\prime}$. This implies together with (**) that the Fourier transform of $\omega\left(A^{*} U(x) A U(-x)\right)$ is contained in

$$
-\operatorname{supp} . \Psi+\overline{\mathscr{V}}+
$$

Since equation (*) is true for arbitrary $\Psi$ we get

$$
\mathscr{F}\left\{\omega\left(A^{*} U(x) A U(x)\right)\right\}(p) \subset \bigcap_{\Psi}-\operatorname{supp} . \Psi+\overline{\mathscr{V}}+=\overline{\mathscr{V}}+
$$

* In the terminology of Schwartz [11], $\mathscr{D}$ is the space of $C^{\infty}$-functions with compact support (Chap I, $\S 2$ ), $\mathscr{S}$ is the space of strongly decreasing $C^{\infty}$-functions (Chap. VII, $\S 3$ ), and $\mathscr{S}^{\prime}$ is the space of tempered distributions (Chap. VII, $\S 4$ ). 
At this point we have used the assumption that there are always states whose support is arbitrarily close to the boundary of the forward lightcone. But this can be proved and we will do this in

Lemma 9. The smallest convex closed cone containing the spectrum coincides with the forward light cone.

Proof. Let us consider two elements $A, B \in \mathfrak{R}(\mathcal{O})$ such that $A(x)=U(x) A U(-x)$ does not commute with $B$ for all $x$. Then there must be vectors $\Psi$ and $\Phi$ with compact support such that the expectation value $(\Phi,[A(x), B] \Psi)=f(x)$ does not vanish. Let $\mathscr{C}$ be the smallest convex closed cone containing the spectrum. Now the Fourier transform $\tilde{f}(p)$ of $f(x)$ has its support contained in $(\operatorname{supp} \Phi-\mathscr{C}) \cup(-\operatorname{supp} \Psi+\mathscr{C})$. But as a consequence of the support of $f(x)$ in $x$-space $f(p)$ can be written as the difference of two functions $f^{+}(p)$ and $f^{-}(p)$ with $f^{+}$holomorphic in the forward tube and $f^{-}$holomorphic in the backward tube.

If now $\mathscr{C}$ is a proper subcone of $\overline{\mathscr{V}}^{+}$, it follows from the double-cone theorem [12] that $f(p)$ vanishes identically. This contradicts our assumptions and hence Lemma 9 holds.

We collect the results of this section in

Theorem 10. In the vacuum theory defined by the functional $\omega$ of Corollary 2 there exists a unitary representation of the translation group which fulfils the spectrum condition.

We have shown in the special case where $\mathfrak{R}(\mathscr{M})$ is a factor that the functional $\omega(A)$ defined in Corollary 2 fulfils the spectrum condition. What about the general case? In order to handle this, we need first some notations. The map $L$ from $\mathfrak{A}(\mathscr{M})$ into the center is a linear positive map. The left kernel of $\mathfrak{A}(\mathscr{M})$ is the set of all $A \in \mathfrak{A}(\mathscr{M})$ such that $L\left(A^{*} A\right)=0$. Since $L$ is a positive map and is continuous in the norm topology, the left kernel is a norm-closed left ideal. We define now:

Definition 11. A positive, linear, invariant map $L$ of $\mathfrak{A}(\mathscr{M})$ into $\mathfrak{S}(\mathscr{M})$ is said to fulfil the spectrum condition, if for every $A \in \mathfrak{A}(\mathscr{M})$ and every test-function $f(x) \in \mathscr{S}$ with support $f(p)$ contained in the complement of $\overline{\mathscr{V}}+$, the operator

$$
\int f(x) U(x) A U^{-1}(x) d^{4} x
$$

is in the left kernel of $L$.

Theorem 12. The map $L$ defined in Theorem 1 fulfils the spectrum condition.

Proof. When we make a direct-integral decomposition of the Hilbert space and the algebra with respect to the center, then the algebras $\mathfrak{A}_{\lambda}(\mathscr{M})$ fulfil $\mu$-almost everywhere the conditions of Theorem 10 (since the center is pointwise invariant). Hence $\int f(x) U_{\lambda}(x) A_{\lambda} U_{\lambda}^{-1}(x) d^{4} x$ is for support $f(p)$ outside the closed forward light-cone $\mu$-almost everywhere in the left kernel of $L_{\lambda}$. This implies $\int d \mu(\lambda) \int f(x) U_{\lambda}(x) \times$ $\times A_{\lambda} U_{\lambda}^{-1}(x) d^{4} x=\int f(x) U(x) A U^{-1}(x) d^{4} x$ is in the left kernel of $L$. 


\section{Extension of the mapping to the local v. Neumann algebras}

We consider again the linear positive map $L(B)$ from $\mathfrak{A}(\mathscr{M})$ into the center of $\mathfrak{R}(\mathscr{M})$. We want to prove the following

Theorem 13. Let $\mathscr{V}$ be any open region (not necessarily bounded) such that there exists a bounded open region $\mathcal{O}$ which is spacelike with respect to $\mathscr{V}$. Define $\mathfrak{A}(\mathscr{V})$ as the smallest norm closed algebra which contains all $\mathfrak{A}\left(\mathcal{O}_{1}\right)$ with $\mathscr{O}_{1} \subset \mathscr{V}$. Let $L$ be the map from $\mathfrak{A}(\mathscr{M})$ to $\mathfrak{S}(\mathscr{M})$ defined in Theorem 1 . Then $L$ restricted to $\mathfrak{A}(\mathscr{V})$ is continuous in the strong operator topology.

The proof of this theorem is essentially based on the " $B, T$-Lemma" ([13], Lemma 9.1.1-9.2.1; [8], Chap. III, § 1, Lemma 2) and proceeds in several steps.

Lemma 14. Let $\Psi$ be a vector which is analytic for the energy and separating for the center $\mathfrak{C}(\mathscr{M})$. Then this vector is cyclic for the ring generated by $\mathfrak{R}(\mathcal{O})$ and $\mathfrak{R}(\mathscr{M})^{\prime} \equiv \mathfrak{R}\left\{\mathfrak{R}(\mathcal{O}), \mathfrak{R}(\mathscr{M})^{\prime}\right\}$. Let $\left\{\Phi_{n}\right\}_{n=1}^{\infty}$ be a sequence such that $\Sigma\left\|\Phi_{n}\right\|^{2}<\infty$. Then there exists a vector $\chi \in \mathfrak{G}$ and a sequence $\left\{T^{n}\right\}_{n=1}^{\infty} \in \mathfrak{R}\left\{\mathfrak{R}(\mathcal{O}), \mathfrak{R}(\mathscr{M})^{\prime}\right\}$ such that

1) $T^{n} \chi=\Phi_{n}$,

2) $\left\|T^{n}\right\| \leqq M$.

Proof. Consider the Hilbertspace $\hat{\mathfrak{F}}=l_{2} \times \mathfrak{G}$ and the ring

$$
\widehat{\mathfrak{R}}=\mathfrak{S} \times \mathfrak{R}\left\{\mathfrak{R}(\mathcal{O}), \mathfrak{R}(\mathscr{M})^{\prime}\right\}
$$

where $\mathfrak{S}$ denotes the ring of all diagonal matrices in $l_{2}$ (with respect to the natural basis). Since $\Psi$ is a cyclic vector for $\mathfrak{R}\left\{\mathfrak{R}(\mathcal{O}), \mathfrak{R}(\mathscr{M})^{\prime}\right\}$, the vector $\hat{\Psi}=\left\{\frac{1}{n} \Psi\right\}$ is cyclic for $\widehat{\mathfrak{R}}$ in $\hat{\mathfrak{G}}$. Now $\left\{\Phi_{n}\right\}=\Phi$ is a vector in $\hat{\mathfrak{G}}$. Since $\Psi$ is cyclic for $\widehat{\mathfrak{R}}$ we can find operators $\hat{A}_{k}=\left\{A_{k}^{n}\right\}$ in $\mathfrak{R}$ such that

$$
\left\|\hat{A}_{k} \hat{\Psi}-\Phi\right\| \leqq 4^{-k}\{\hat{\Psi} \| \text {. }
$$

Now the set of vectors $\chi \in \mathfrak{G}$ for which

$$
\sum_{n, k}\left\|\frac{2^{k}}{n}\left(A_{k+1}^{n}-A_{k}^{n}\right) \chi\right\|^{2}<\infty
$$

is not empty. In particular $\Psi$ is in this set. From a well known theorem ([8], Chap. III, $\S 1$, Lemma 2) follows the existence of an operator $B \in \mathfrak{R}\left\{\mathfrak{R}(\mathcal{O}), \mathfrak{R}(\mathscr{M})^{\prime}\right\}$ with $\Psi$ in the range of $B,\|B\| \leqq 1$, and $\left\|\frac{2^{k}}{n}\left(A_{k+1}^{n}-A_{k}^{n}\right) B\right\| \leqq 1$.

This implies $\frac{1}{n} A_{k}^{n} B$ converges to $T^{n}$ with $\left\|T^{n}\right\| \leqq M$.

On the other hand let $\chi$ be the vector such that $B \chi=\Psi$; then we have

$$
T^{n} \chi=\lim _{k \rightarrow \infty} \frac{1}{n} A_{k}^{n} B \chi=\lim _{k \rightarrow \infty} A_{k}^{n} \frac{1}{n} \Psi=\Phi_{n}, \quad \text { q.e.d. }
$$


Lemma 15. Let $\Phi(z)$ be a holomorphic function with values in the Hilbert space $\mathfrak{F}$ for $|z|<1$. Assume $\mu(\theta)$ is a positive measure on the unit circle such that $d \mu(\theta) \geqq d \theta$. Assume moreover that $\lim _{r \rightarrow 1} \int\left\|\Phi\left(r e^{i \theta}\right)\right\|^{2}$ $d \mu(\theta)<\infty$.

Then in $\mathfrak{R}\left\{\mathfrak{R}(\mathcal{O}), \mathfrak{R}(\mathscr{M})^{\prime}\right\}$ there exists an operator-valued function $T(z)$ holomorphic in $|z|<1$ and a vector $\chi \in \mathfrak{F}$ such that

and

$$
T(z) \chi=\Phi(z)
$$

$$
\lim _{r \rightarrow 1}\left\|\int T^{*}(z) T(z) d \mu(\theta)\right\|<\infty .
$$

Proof. Since $\Phi(z)$ is an analytic function for $|z|<1$ we can write

Now we have

$$
\Phi(z)=\sum \Phi_{n} z^{n}
$$

$$
\sum\left\|\Phi_{n}\right\|^{2}=\lim _{r \rightarrow 1} \int\|\Phi(z)\|^{2} d \theta \leqq \lim _{r \rightarrow 1} \int\|\Phi(z)\|^{2} d \mu(\theta)<\infty .
$$

Hence we have by Lemma $11 \chi_{1} \in \mathfrak{S}$ and $T_{n} \in \mathfrak{R}\left\{\mathfrak{R}(\mathcal{O}), \mathfrak{R}(\mathscr{M})^{\prime}\{\right.$ with $\left\|T_{n}\right\| \leqq 1$ such that

$$
T_{n} \chi_{1}=\Phi_{n}, \text { i.e. } \sum T_{n} z^{n} \chi_{1}=\Phi(z) .
$$

Since $\left\|T_{n}\right\| \leqq 1$ we have

$$
T^{\prime}(z)=\sum T_{n} z^{n} \quad \text { is holomorphic for } \quad|z|<1 .
$$

Consider now in $\mathfrak{G}$ the subset $\mathfrak{H}_{0}$ of vectors such that

$$
\lim _{r \rightarrow 1} \int\left\|T^{\prime}(z) \Psi\right\|^{2} d \mu(\theta)+\|\Psi\|^{2}<\infty .
$$

Clearly $\chi_{1} \in \mathfrak{H}_{0}$.

The expression $(*)$ makes $\mathfrak{G}_{0}$ a Hilbert space.

Since $\mathfrak{G}_{0}$ is invariant under all unitary operators in $\mathfrak{R}\left\{\mathfrak{R}(\mathcal{O}), \mathfrak{R}(\mathscr{M})^{\prime}\right\}^{\prime}$, we can find a bounded operator $B \in \mathfrak{R}\left\{\mathfrak{R}(\mathcal{O}), \mathfrak{R}(\mathscr{M})^{\prime}\right\}$ whose range is exactly $\mathfrak{G}_{0}$ (for proof see [13], Lemma 9.1.3, 9.1.4).

Define now $T(z)=T^{\prime}(z) \cdot B$. Then $T(z)$ is again holomorphic in $|z|<1$. Moreover we have for all $\chi \in \mathfrak{F}$

and hence

$$
\int\|T(z) \Psi\|^{2} d \mu(\theta) \leqq M\|\Psi\|^{2}
$$

$$
\left\|\int T^{*}(z) T(z) d \mu(\theta)\right\| \leqq M, \quad \text { q.e.d. }
$$

Lemma 16. Let $\Psi$ be a vector analytic for the energy and $a$ a spacelike vector. This implies $U(\lambda a) \Psi$ is holomorphic in $\lambda$ in some strip $|\operatorname{Im} \lambda|<b$. Then there exists in $\mathfrak{R}\left\{\mathfrak{R}(\mathcal{O}), \mathfrak{R}(\mathscr{M})^{\prime}\right\}$ and operator-valued function $T(\lambda)$ holomorphic in $|\operatorname{Im} \lambda|<b_{1}<b$ and bounded for real $\lambda$. Moreover there exists a vector $\chi \in \mathfrak{S}$ such that

$$
T(\lambda) \chi=U(\lambda a) \Psi \text { for }|\operatorname{Im} \lambda|<b_{1} .
$$

Proof. Since $U(\lambda a)$ is unitary for real $\lambda$ we have $\|U(\lambda a) \Psi\|<M$ for $|\operatorname{Im} \lambda| \leqq b_{1}<b$. Let us now make a conformal mapping such that 
the image of $|\operatorname{Im} \lambda|<b_{1}$ is $|z|<1$ and the image of $\operatorname{Im} \lambda=0$ is $\operatorname{Im} z=0$. Then $\Phi(z)=U(\lambda a) \Psi$ is bounded in $|z| \leqq 1$. Thus $\Phi(z)$ fulfils the condition of Lemma 14 for the measure $d \mu(\theta)=d \theta(1+\delta(\theta)+\delta(\theta-\pi))$. This implies the existence of $T(z) \in \mathfrak{R}\left\{\mathfrak{R}(\mathcal{O}), \mathfrak{R}(\mathscr{M})^{\prime}\right\}$ and $\chi \in \mathfrak{F}$ with

and

$$
T(z) \chi=\Phi(z)
$$

$$
\lim _{r \rightarrow 1}\left\|\int T^{*}(z) T(z) d \theta+T^{*}(r) T(r)+T^{*}(-r) T(-r)\right\|<M .
$$

Since $T(z)$ is bounded for $|z|<1$ it follows that $\|T(x)\| \leqq M_{1},|x|<1$. Transforming back to the variable $\lambda$ we the desired result.

Proof of Theorem 13. Assume we have chosen for $\Psi$ in Lemma 16 a vector which is analytic for the energy and separating for the center, and for $\mathcal{O}$ a region which is spacelike to $\mathscr{V}$. Then the operators $T\left(\lambda_{n}\right)$ of Lemma 16 commute with $\mathfrak{A}(\mathscr{V})$. Let now $A_{n} \in \mathfrak{A}(\mathscr{V})$ with $\left\|A_{n}\right\| \leqq 1$ and assume the $A_{n}$ converge to zero in the strong operator topology. Let $L\left(A_{n}\right)$ be the image of $A_{n}$ under the map described in Theorem 1 . Then we have

$$
\begin{aligned}
\left|\left(\Phi, L\left(A_{n}\right) \Psi\right)\right| & \leqq \lim _{j \rightarrow \infty}\left|\left(U\left(-\lambda_{j} a\right) \Phi, A_{n} U\left(-\lambda_{j} a\right) \Psi\right)\right| \\
& =\lim _{j \rightarrow \infty}\left|\left(U\left(-\lambda_{j} a\right) \Phi, A_{n} T\left(-\lambda_{j}\right) \chi\right)\right| \\
& =\lim _{j \rightarrow \infty}\left|\left(U\left(-\lambda_{j} a\right) \Phi, T\left(-\lambda_{j}\right) A_{n} \chi\right)\right| \leqq\|\Phi\| \sup _{j}\left\|T\left(-\lambda_{j}\right)\right\|\left\|A_{n} \chi\right\| .
\end{aligned}
$$

Since $\left\|T\left(\lambda_{j}\right)\right\|$ is bounded we have

$$
\lim _{n \rightarrow \infty} L\left(A_{n}\right) \Psi=0
$$

and since $\Psi$ is separating for the center and

$$
\left\|L\left(A_{n}\right)\right\| \leqq\left\|A_{n}\right\| \leqq 1,
$$

it follows ([8], Chap. I, $\S 4$, subsection 6, Prop. 4) that the operators $L\left(A_{n}\right)$ converge to zero in the strong operator topology, q.e.d.

Theorem 17. The map $L$ from $\mathfrak{R}(\mathscr{V})$ into $\mathfrak{E}(\mathscr{M})$ is normal ([8], Chap. I, §4, subsection 3, Def. 2).

Proof. Since $\Psi$ is separating for the center it is sufficient to show that the linear functional

$$
(\Psi, L(A) \Psi) \text { is normal on } \mathfrak{R}(\mathscr{V}) .
$$

For every $A \in \mathfrak{R}(\mathscr{V})$ the sequence

$$
\left(T^{*}\left(-\lambda_{j}\right) U\left(-\lambda_{j} a\right) \Psi, A \chi\right) \rightarrow(\Psi, L(A) \Psi) \text { as } j \rightarrow \infty,
$$

and the limit is linear in $A$.

Since the sequence $\left(T^{*}\left(-\lambda_{n}\right) U\left(-\lambda_{n} a\right) \Psi\right)$ is bounded, there exists a vector $\Phi$ in $\overline{\mathfrak{R}(\mathscr{V}) \chi}$ with

$$
\Phi=\text { weak } \lim _{n^{\prime} \rightarrow \infty} E\left(T^{*}\left(-\lambda_{n^{\prime}}\right) U\left(-\lambda_{n^{\prime}} a\right) \Psi\right)
$$


with $E \mathfrak{Y}=\overline{\mathfrak{R}(\mathscr{V}) \chi}$. This implies

$$
(\Psi, L(A) \Psi)=(\Phi, A \chi) \text { is normal, }
$$

q.e.d.

\section{On physical equivalence and local unitary equivalence}

For the representation induced by a positive linear functional, the maximal two-sided ideal contained in the null space of this functional is of special interest. If this ideal contains only zero, then the representation will be faithful.

Theorem 18. The null space of the map $L(A)$ from $\mathfrak{R}(\mathscr{V})$ into $\mathfrak{C}(\mathscr{M})$ contains no two-sided ideal. The null space of $L$ as a map from $\mathfrak{A}(\mathscr{M})$ into $\mathfrak{E}(\mathscr{M})$ contains also no two-sided ideal.

Proof. Let $\Psi$ be again the separating vector for the center used in the last section. Then for any $A \in \mathfrak{R}^{+}(\mathscr{V})\left(\mathfrak{R}^{+}=\right.$positive elements of $\left.\mathfrak{R}\right)$ we have

$$
L(A)=0 \quad \text { if and only if }(\Psi, L(A) \Psi)=0 .
$$

$(\Psi, L(A) \Psi)$ is a positive linear functional on the algebra $\mathfrak{A}(\mathscr{M})$. We look now at the standard representation of $\mathfrak{A}(\mathscr{M})$ given by this functional. If we call the representation space $\Re$, then this representation can be considered as a homomorphism $\varphi$ of $\mathfrak{A}(\mathscr{M})$ into $\mathscr{L}(\Re)$, the space of bounded linear operators in $\mathfrak{R}$ :

$$
\mathfrak{A}(\mathscr{M}) \stackrel{\Phi}{\rightarrow} \mathscr{L}(\Re) .
$$

We look at the kernel of the homomorphism $\varphi$, Ker $\varphi$, which is a twosided ideal in $\mathfrak{A}(\mathscr{M})$. Since $L(\mathbf{1})=\mathbf{1}$ it follows that $\varphi$ is not the null homomorphism, i.e. that Ker $\varphi$ is a proper two-sided ideal in $\mathscr{A}(\mathscr{M})$.

Let $\mathscr{V}$ now be any open region described in Theorem 13 and $\varphi_{v}$ the restriction of $\varphi$ to $\mathfrak{A}(\mathscr{V})$. In this case we know from Theorem 13 that $\varphi_{v}$ is continuous in the strong operator topology and extends therefore to $\mathfrak{R}(\mathscr{V})$. Now $\operatorname{Ker} \varphi_{v}$ is a two-sided ideal in $\mathfrak{R}(\mathscr{V})$ closed in the strong operator topology.

From this it follows ([8], Chap. I, $\S 4$, subsection 4, Cor. 3) that there exists a projection $E_{v}$ in the center of $\mathfrak{R}(\mathscr{V})$ such that

$$
\operatorname{Ker} \varphi_{v}=\mathfrak{R}(\mathscr{V}) \cdot E_{v}, E_{v} \in \mathfrak{C}(\mathscr{V}) .
$$

Let $\mathscr{V}^{\prime}$ be a region such that $\mathscr{V}^{\prime}+x \subset \mathscr{V}$ for $x$ from a certain neighbourhood of the origin. Then we get

$$
\operatorname{Ker} \varphi_{v^{\prime}}=\mathfrak{R}\left(\mathscr{V}^{\prime}\right) \cdot E_{v^{\prime}}, E_{v^{\prime}} \in \mathfrak{S}\left(\mathscr{V}^{\prime}\right) .
$$

This implies $\mathfrak{R}\left(\mathscr{V}^{\prime}\right) E_{v}=\mathfrak{R}\left(\mathscr{V}^{\prime}\right) E_{v^{\prime}}$ and thus, since 1 is in $\mathfrak{R}\left(\mathscr{V}^{\prime}\right)$, we have

Using Theorem 8 we get

$$
E_{v}=E_{v^{\prime}} .
$$

$$
E_{v}=E_{v^{\prime}} \in \mathfrak{S}(\mathscr{M}) .
$$


Comparing now all possible open regions we find: there exists a projection $E$ in $\mathfrak{夭}(\mathscr{M})$ such that

$$
\mathfrak{A}(\mathcal{O}) \cap \operatorname{Ker} \varphi=\mathfrak{R}(\mathscr{M}) \cdot E \cap \mathfrak{X}(\mathcal{O}) \quad \text { for all } \mathcal{O} .
$$

Let us now choose a vector $\Psi$ such that $E \Psi=\Psi$. Then we have by Theorem 1

$$
(\Psi L(A), \Psi)=\lim _{n \rightarrow \infty}\left(U\left(-\lambda_{n} \alpha\right) \Psi, A U\left(-\lambda_{n} \alpha\right) \Psi\right) .
$$

Putting $A=E$ we find $L(E)=0$ only if $E=0$. This implies

$$
\operatorname{Ker} \varphi \cap \mathfrak{A}(\mathcal{O})=0 .
$$

Since $\varphi$ is norm-continuous and $\mathfrak{A}(\mathscr{M})$ is generated by all $\mathfrak{A}(\mathcal{O})$ we find

This proves the theorem ${ }^{\star}$.

$$
\operatorname{Ker} \varphi=0 \text {. }
$$

If we now make more restrictive assumptions we get:

Corollary 19. If $\Re(\mathscr{M})$ is a factor, then by Corollary $2 L(A)=1 \cdot \omega(A)$ where $\omega(A)$ is a positive functional. The canonical representation $\varphi$ of $\omega(A)$ is a faithful representation of the norm closed ring $\mathfrak{A}(\mathscr{M})$. For every open region $\mathscr{V}$ as in Theorem $13 \varphi$ extends to $\mathfrak{R}(\mathscr{V})$ and $\varphi(\mathfrak{R}(\mathscr{V}))$ is a von Neumann algebra.

Proof. From Theorem 18 it follows that $\varphi(\mathfrak{A}(\mathscr{M}))$ is faithful. Since by virtue of Theorem $17 L(A)$ is a normal map of $\mathfrak{R}(\mathscr{V})$ into $\mathfrak{E}(\mathscr{M})$, $\omega(A)$ is a normal, linear positive functional. But this implies ([8], Chap. $I, \S 4$, subsection 3, Prop. 1) that $\varphi$ is normal and therefore $\varphi(\mathfrak{R}(\mathscr{V}))$ is a v. Neumann algebra ([8] Chap. I, §4, subsection 3, Cor. 2), q.e.d.

Corollary 20. If $\mathfrak{R}(\mathscr{M})=\mathscr{L}(\mathfrak{F})$, the ring of all bounded operators, then the map

$$
\mathfrak{R}(\mathscr{V}) \rightarrow \varphi(\mathfrak{R}(\mathscr{V}))
$$

is unitarily implementable for every open region $\mathscr{V}$ described in Theorem 13.

Proof. Since $\mathfrak{R}(\mathscr{M})=\mathscr{L}(\mathfrak{S})$ contains every vector which is analytic for the energy, there exists a cyclic and separating vector for $\mathfrak{R}(\mathscr{V})$ (Lemma 5). Since $\varphi(\mathscr{H}(\mathscr{M}))$ is a cyclic representation and $\Omega \in \mathfrak{R}$ is an invariant vector, we have that $\varphi(\mathscr{A}(\mathscr{M})) \Omega$ is dense in $\Omega$, and hence $\Omega$ is cyclic and separating for $\varphi(\mathfrak{R}(\mathscr{V}))$. Since now $\varphi(\mathfrak{R}(\mathscr{V}))$ is a von Neumann algebra we have that $\varphi(\mathfrak{R}(\mathscr{V}))$ is unitarily implementable ([8], Chap. III, §1, subsection 4, Th. 3), q.e.d.

* $\varphi$ restricted to $\mathfrak{H}(\mathcal{O})$ is an isomorphism and $\varphi$ and $\varphi^{-1}$ are continuous. Hence $\varphi$ extends to the norm-closure of $\cup \mathfrak{U}(\mathcal{O})$ and $\varphi^{-1}$ extends to the norm-closure of $\cup \varphi(\mathfrak{A}(\mathcal{O}))$. This implies $\varphi$ is an isomorphism for $\mathfrak{A}(\mathscr{M})$. 


\section{On the uniqueness of the vacuum theory}

We consider the following set of functionals on $\mathscr{A}(\mathscr{M}): \omega$ is an element of $\mathfrak{V}$ if it has the following properties:

1) $\omega$ is a positive linear functional on $\mathfrak{A}(\mathscr{M})$.

2) $\omega$ is translation-invariant.

3) $\omega$ fulfils the spectrum condition.

We want to prove the following

Theorem 21. Let $\omega_{1}$ and $\omega_{2}$ be elements from $\mathfrak{V}$. Denote by $\varphi_{1}$ and $\varphi_{2}$ the canonical representations of $\omega_{1}$ and $\omega_{2}$. Assume that for $\mathcal{O}$ an open bounded region $\varphi_{1}(\mathfrak{A}(\mathcal{O}))$ and $\varphi_{2}(\mathfrak{A}(\mathcal{O}))$ are equally faithful representations of $\mathscr{A}(\mathcal{O})$. Assume, moreover, that the rings generated by $\left\{\varphi_{1}(\mathfrak{H}(\mathcal{O}))\right.$, $\left.\mathfrak{夭}_{1}(\mathscr{M})\right\}$ and by $\left\{\varphi_{2}(\mathscr{H}(\mathcal{O})), \mathfrak{\complement}_{2}(\mathscr{M})\right\}\left(\mathfrak{G}_{1}(\mathscr{M})\right.$ resp. $\mathfrak{\complement}_{2}(\mathscr{M})$ are the centers of the global v. Neumann algebras) are unitarily equivalent. In this case $\varphi_{1}(\mathfrak{A}(\mathscr{M}))$ and $\varphi_{2}(\mathfrak{A}(\mathscr{M}))$ are unitarily equivalent. Moreover, if $\omega_{1}\left(C_{1}\right)=\omega_{2}\left(C_{2}\right)$ for every element form the center, then $\omega_{1}=\omega_{2}$.

The proof of this theorem will be made in several steps. We start with the notation.

Let us denote by $\mathfrak{G}_{1}$ and $\mathfrak{G}_{2}$ the representation space of $\varphi_{1}$ and $\varphi_{2}$. Let $U_{1}(x)$ and $U_{2}(x)$ be the representations of the translation group in $\mathfrak{G}_{1}$ and $\mathfrak{G}_{2}$, and let $\Omega_{1}$ and $\Omega_{2}$ be the cyclic vacuum states in $\mathfrak{G}_{1}$ and $\mathfrak{G}_{2}$. We set $\hat{\mathfrak{G}}=$ the direct sum of $\mathfrak{G}_{1}$ and $\mathfrak{G}_{2}$,

$$
\begin{aligned}
\hat{U}(x) & =\left(\begin{array}{lr}
U_{1}(x) & 0 \\
0 & U_{2}(x)
\end{array}\right), \\
\hat{A} & =\left(\begin{array}{lr}
\varphi_{1}(A) & 0 \\
0 & \varphi_{2}(A)
\end{array}\right), \\
\hat{\mathfrak{A}}(\mathcal{O}) & =\{\hat{A} \mid A \in \mathfrak{A}(\mathcal{O})\},
\end{aligned}
$$

and $\hat{\mathfrak{R}}(\mathcal{O})=$ the strong closure of $\hat{\mathfrak{A}}(\mathcal{O})$.

Let us denote by $P_{0}$ the projections onto all states in $\hat{\mathfrak{H}}$ which are invariant under the action of $\hat{U}(x)$.

Lemma 22. Let $B$ be from $\hat{\mathfrak{R}}(\mathcal{O})^{\prime}$; then there exists an element $B_{0}$ with the properties

1) $B_{0} \in \hat{\mathfrak{R}}(\mathscr{M})^{\prime}$.

2) $U(x) B_{0} U^{-1}(x)=B_{0}$ for all $x$.

3) $P_{0} B P_{0}-P_{0} B_{0} P_{0}=0$.

Proof. Let $\mathcal{O}_{1} \subset \mathcal{O}$ such that $\mathcal{O}_{1}+x \subset \mathcal{O}$ for a suitable neighbourhood $\mathscr{N}$ of the origin.

We define an operator $B_{0}$ by the relation

$$
B_{0} \sum_{i} A_{i} P_{0} \Psi_{i}=\sum A_{i} P_{0} B P_{0} \Psi_{i} \text { for all } A_{i} \in \hat{\mathfrak{R}}\left(\mathcal{O}_{1}\right)
$$

By Lemma 5 we have that $P_{0} \hat{\mathfrak{S}}$ is a cyclic subspace for $\hat{\mathfrak{R}}\left(\mathcal{O}_{1}\right)$. This means $B_{0}$ is densely defined. $B_{0}$ is clearly linear. We have to show that $B_{0}$ is single-valued. 
Assume

$$
\sum A_{i} P_{0} \Psi_{i}=0, \quad A_{i} \in \mathcal{O}_{1}
$$

Then we have for $x \in \mathscr{N}$

$\sum U(x) A_{i} U^{-1}(x) B P_{0} \Psi_{i}=0$, and hence $\sum A_{i} U^{-1}(x) B P_{0} \Psi_{i}=0$.

The last expression, however, is, by the spectrum condition, the boundary value of an analytic function. So we get by analytic continuation

$$
\sum A_{i} U(-x+i y) B P_{0} \Psi_{i}=0 \text { for all } y \in \mathscr{V}+
$$

Taking now the limit $y^{0} \rightarrow \infty$, we get

$$
0=\sum A_{i} P_{0} B P_{0} \Psi=B_{0} \sum A_{i} P_{0} \Psi_{i}
$$

$B_{0}$ commutes with all operators of $\mathcal{O}_{1}$ because

$$
A B_{0} \sum A_{i} P_{0} \Psi_{i}=A \sum A_{i} P_{0} B P_{0} \Psi_{i}=B_{0} A \sum A_{i} P_{0} \Psi_{i}
$$

Assume now $B$ is positive; then $P_{0} B P_{0}$ is positive. Hence

$$
\begin{gathered}
\left(\sum A_{i} P_{0} \Psi_{i}, B_{0} \sum A_{j} P_{0} \Psi_{\jmath}\right) \\
=\left(\sum A_{i}\left(P_{0} B P_{0}\right)^{\frac{1}{2}} \Psi_{i}, \sum A_{i}\left(P_{0} B P_{0}\right)^{\frac{1}{2}} \Psi_{i}\right) \geqq 0 .
\end{gathered}
$$

At this point we have used the fact that $B$ commutes with $\mathfrak{R}\left(\mathcal{O}_{1}\right)$. Using large translations we find that $P_{0} B P_{0}$ commutes with $P_{0} \mathfrak{R}\left(\mathcal{O}_{1}\right) P_{0}([4],[14])$.

This implies if $B \geqq 0$, then $B_{0} \geqq 0$, or equivalently, if $B$ is selfadjoint, then $B_{0}$ is also, and the upper and lower bounds of $B_{0}$ are majorized by the upper and lower bounds of $B$. Thus $\left\|B_{0}\right\|<\|B\|$.

Finally, since $B_{0}$ maps the vacuum space into itself, we have for $x \in \mathscr{N}$ and $A_{i} \in \mathcal{O}_{1}$

$$
\begin{aligned}
U(x) B_{0} U(-x) \sum A_{i} P_{0} \Psi_{i} & =\sum A_{i} U(x) B_{0} U(-x) P_{0} \Psi_{i} \\
& =\sum A_{i} B_{0} P_{0} \Psi_{i}=B_{0} \sum A_{i} P_{0} \Psi_{i} .
\end{aligned}
$$

This implies $B_{0}$ commutes with the translation group and hence

$$
B_{0} \in \hat{\mathfrak{R}}(\mathscr{M})^{\prime}, \quad \text { q.e.d. }
$$

Proof of Theorem 21. Let $V$ be the unitary operator mapping $\mathfrak{G}_{1}$ into $\mathfrak{H}_{2}$ and having the property

$$
V \varphi_{1}(A)=\varphi_{\mathbf{2}}(A) V \quad \text { for all } \quad A \in \mathfrak{A}(\mathcal{O})
$$

$V$ is, of course, not uniquely defined by this relation and we can change $V$ by left-multiplication with any unitary operator from $\left\{\varphi_{\mathbf{2}}(\mathfrak{A}(\mathcal{O}))\right.$, $\left.\mathfrak{E}_{2}(\mathscr{M})\right\}^{\prime}$. Since $\Omega_{2}$ is a cyclic vector for this ring we can choose $V$ such that

$$
\left(\Omega_{2}, V \Omega_{1}\right) \neq 0
$$


Consider in $\mathfrak{G}_{1} \oplus \mathfrak{G}_{2}$ the operator

$$
\hat{V}=\left(\begin{array}{rr}
0 & V^{-1} \\
V & 0
\end{array}\right) .
$$

As a consequence of relation $(*)$ we have

$$
\hat{A} \hat{V}=\hat{V} \hat{A} \text { for all } \hat{V} \in \hat{\mathscr{A}}(\mathcal{O}) \text {. }
$$

We consider now the operator $\hat{V}_{0}$ (see Lemma 19).

$\hat{V}_{0}$ commutes with $\hat{\mathcal{Y}}(\mathscr{M})$ and $\left(\Omega_{2}, \hat{V}_{0} \Omega_{1}\right) \neq 0$ by equation (**). From the construction of $\hat{V}_{0}$ it follows that

$$
\hat{V}_{0}=\left(\begin{array}{lr}
0 & \left(V^{-1}\right)_{0} \\
V_{0} & 0
\end{array}\right) \quad \text { with } \quad V_{0} \neq 0 .
$$

This implies the relation

$$
V_{0} \varphi_{1}(A)=\varphi_{2}(A) V_{0} \text { for all } A \in \mathfrak{A}(\mathscr{M}) .
$$

Since $\varphi_{1}$ resp. $\varphi_{3}$ are cyclic representations it follows by a result of H. ARAKI [4] that the commutant of $\varphi_{1}(\mathcal{A}(\mathscr{M}))$ resp. $\varphi_{2}(\mathfrak{A}(\mathscr{M}))$ is identical with the center of $\Re_{1}(\mathscr{M})$ resp. $R_{2}(\mathscr{M})$. If $E$ is the support of $V_{0}$ and $F$ the range of $V_{0}$ then $E$ resp. $F$ are from the center of $\Re_{1}(\mathscr{M})$ resp. $\mathfrak{R}_{2}(\mathscr{M})$. This implies by the generalized Schur's lemma (e.g. [6], $\S 21)$

$$
E \varphi_{1}(\mathscr{U}(\mathscr{M})) \text { is unitarily equivalent to } F \varphi_{2}(\mathfrak{A}(\mathscr{M})) .
$$

We have to show that $V$ can be chosen such that $E$ and $F$ are the identity. Let $P_{0}^{1}$ resp. $P_{0}^{2}$ be the projections onto the invariant subspaces in $\mathfrak{G}_{1}$ resp. $\mathfrak{S}_{2} . P_{0}^{1} \mathfrak{G}_{1}$ resp. $P_{0}^{2} \mathfrak{G}_{2}$ are generated from the cyclic vacua $\Omega_{1}$ resp. $\Omega_{2}$ by the center $\mathfrak{夭}_{1}(\mathscr{M})$ resp. $\mathfrak{夭}_{2}(\mathscr{M})$.

From the assumption that $V$ maps not only $\varphi_{2}(\mathfrak{A}(\mathcal{O}))$ onto $\varphi_{1}(\mathcal{O})$ but also $\mathfrak{C}_{2}(\mathscr{M})$ onto $\mathfrak{S}_{1}(\mathscr{M})$ it follows that

Hence

$$
V P_{0}^{1} \mathfrak{G}_{1}=\overline{V \mathfrak{G}_{1}(\mathscr{M}) \Omega_{1}}=\overline{\mathfrak{S}_{2}(\mathscr{M}) V \Omega_{1}} .
$$

$$
P_{0}^{2} V P_{0}^{1} \mathfrak{S}_{1}=\overline{\mathfrak{S}_{2}(\mathscr{M}) P_{0}^{2} V \Omega_{1}} .
$$

Let now $F$ be the minimal projection in $\mathfrak{F}_{2}(\mathscr{M})$ with

$$
F P_{0}^{2} V \Omega_{1}=P_{0}^{2} V \Omega_{1} \text {. }
$$

Then $(\mathbf{1}-F) \Omega_{2}$ is cyclic for the ring $(\mathbf{1}-F)\left\{\varphi(\mathscr{U}(\mathcal{O})), \mathfrak{G}_{2}(\mathscr{M})\right\}^{\prime}$ in $\left(\mathbf{1}-F^{\prime}\right) \mathfrak{G}_{2}$. This means we can find an unitary operator $W$ in this ring such that $\left(\left(\mathbf{1}-F^{\prime}\right) \Omega_{2}, W V \Omega_{1}\right)=0$.

Hence the operator $V^{\prime}=\left(\mathbf{1}-F^{\prime}\right) W \cdot V+F V$ establishes the unitary equivalence and

$$
\overline{\mathfrak{C}_{2}(\mathscr{M}) P_{0}^{2} V^{\prime} \Omega_{1}} \supset \overline{\mathcal{夭}_{2}(\mathscr{M}) P_{0}^{2} V \Omega_{1}} \text {. }
$$

Furthermore the right-hand-side is a proper subspace of the left-handside. This shows that the only upper bound of

$$
\overline{\mathcal{S}_{2}(\mathscr{M}) P_{0}^{2} V \Omega_{1}} \text { is } \overline{\mathcal{S}_{2}(\mathscr{M}) \bar{\Omega}_{1}}=P_{0}^{2} \mathfrak{G}_{2} .
$$


Hence we can find a $V$ such that

$$
\overline{\mathfrak{S}_{2}(\mathscr{M}) P_{0}^{2} V \Omega_{1}}=P_{0}^{2} \mathfrak{G}_{2} \text {. }
$$

Constructing with this operator $V_{0}$ we see that the support and range of $V_{0}$ are the whole Hilbert spaces $\mathfrak{G}_{1}$ resp. $\mathfrak{S}_{2}$. This proves that the two representations $\varphi_{1}$ and $\varphi_{2}$ are unitarily equivalent, and that this equivalence extends to the unitary representations of the translation group.

It remains to show that the two defining functionals $\omega_{1}$ and $\omega_{2}$ are identical.

Let $\Omega_{1}$ be the cyclic vector in $\mathcal{H}_{1}$ and $\Omega_{2}^{\prime}$ be the image of the cyclic vector in $\mathfrak{G}_{2}$ under this unitary map. $\Omega_{2}^{\prime}$ is also an invariant vector. But for each element of the center we have assumed

$$
\left(\Omega_{1}, C \Omega_{1}\right)=\left(\Omega_{2}^{\prime}, C \Omega_{2}^{\prime}\right) \text {. }
$$

Now $P_{0} \mathfrak{G}_{1}$, the space of invariant vectors, is generated by $\mathfrak{E}(\mathscr{M})$ from $\Omega_{1}$ resp. $\Omega_{2}^{\prime}$. This implies the existence of a unitary operator $V$ in $P_{0} \mathfrak{G}_{1}$ which maps $\Omega_{1}$ onto $\Omega_{2}^{\prime}$ and which commutes with $\mathfrak{C}(\mathscr{M})$ restricted to $P_{0} \mathfrak{S}_{1}=\mathfrak{C}(\mathscr{M})_{P_{0}}$. But $\mathfrak{C}(\mathscr{M})_{P_{0}}$ is a maximal Abelian algebra and hence $V \in \mathbb{C}(\mathscr{M})_{P_{0}}$.

This implies $V=P_{0} W P_{0}$ with $W \in \mathfrak{C}(\mathscr{M})$ and $W$ a unitary operator. Hence

$$
\left(\Omega_{2}^{\prime} A \Omega_{2}^{\prime}\right)=\left(W \Omega_{1} A W \Omega_{1}\right)=\left(\Omega_{1} A \Omega_{1}\right) \text { for all } A \in \mathfrak{R}(\mathscr{M}) \text {, q.e.d. }
$$

Theorem 23. Let $\mathfrak{A}(\mathscr{M})=\{\mathfrak{U}(\mathcal{O})\}$ be a local ring system in a Hilbert space $\mathfrak{S}$. $\mathfrak{A}$ norm-closed and $\mathfrak{A}(\mathcal{O}+x)=U(x) \mathfrak{A}(\mathcal{O}) U(-x)$, where $U(x)$ is a unitary representation of the translation group fulfilling the spcetrum condition. Let $\mathfrak{\complement}(\mathscr{M})$ be the center of the v. Neumann algebra generated by $\mathfrak{A}(\mathscr{M})$. Let $L(\mathfrak{A})$ be a linear positive map of $\mathfrak{A}(\mathscr{M})$ into $\mathfrak{S}(\mathscr{M})$ with the properties $\mathfrak{E}(\mathscr{M})$.

1) $L$ is defined on the norm-closed ring generated by $\mathscr{A}(\mathscr{M})$ and

2) $L$ is invariant i.e. $L(U(x) A U(-x))=L(A)$.

3) $L$ fulfils the spectrum condition.

4) For every open region $\mathcal{O}$ the map $L$ is continuous in the strong operator topology on the ring generated by $\mathfrak{A}(\mathcal{O})$ and $\mathfrak{S}(\mathscr{M})$.

5) For $A \in \mathfrak{C}(\mathscr{M}), L(A)=A$.

Then $L$ is identical with the map $L$ defined in Theorem 1 .

Corollary 24. Let $A \in \mathfrak{R}(\mathcal{O})$, where $\mathcal{O}$ is any open bounded region. Then $\lim _{a \rightarrow \infty}(\Psi, U(a) A U(-a) \Psi)$ converges for any spacelike $a$ to the limit $(\Psi, L(A) \Psi)$. This convergence is uniformin the region $\left|a^{0}\right| \leqq \alpha|\vec{a}|$ with $\alpha<1$.

Corollary 25. If there exists in $\mathfrak{G}$ a representation of the inhomogenous Lorentz group such that

$$
U(a, \Lambda) \mathfrak{A}(\mathcal{O}) U^{-1}(a, \Lambda)=\mathfrak{A}(\Lambda \mathcal{O}+a),
$$


then the linear map $L$ of $\mathfrak{A}(\mathscr{M})$ into the center $\mathfrak{S}(\mathscr{M})$ is invariant under the inhomogenous Lorentz group, i.e.

$$
L\left(U(a, \Lambda) A U^{-1}(a, \Lambda)\right)=L(A) .
$$

Proof of Theorem 23. Let $\Psi$ be a fixed vector separating for the center and let $L_{0}$ resp. $L_{1}$ be two maps of $\mathscr{U}(\mathscr{M})$ into $\mathfrak{S}(\mathscr{M})$ fulfilling the conditions 1) -5 ) of Theorem 23. Consider now the functionals $\omega_{0}$ resp. $\omega_{1}$ equal to $\left(\Psi, L_{0}(A) \Psi\right)$ resp. $\left(\Psi, L_{1}(A) \Psi\right)$. Let $D_{0}(A)$ resp. $D_{1}(A)$ be the cyclic representations of $\omega_{0}$ resp. $\omega_{1}$. Since $\Psi$ was chosen to be separating for the center we have by virtue of Theorem 18 that the canonical homomorphisms $\varphi_{0}: A \rightarrow D_{0}(A)$ resp. $\varphi_{1}: A \rightarrow D_{1}(A)$ are isomorphisms. This implies we have an isomorphism $\varphi_{01}: D_{0}(A) \rightarrow D_{1}(A)$. Now Theorem 13 and Theorem 17 say that $\varphi_{0}$ resp. $\mathcal{F}_{1}$ restricted to any bounded open region $\mathcal{O}$ are continuous in the strong operator topology and normal. This implies $\varphi_{01}$ restricted to $\mathcal{O}$ is continuous in the strong topology and normal. Since the representations $D_{0}$ and $D_{1}$ are cyclic representations it follows from the Reeh-Schlieder theorem that $D_{0}(\mathfrak{R}(\mathcal{O}))$ and $D_{1}(\mathfrak{R}(\mathcal{O})$ ) have a cyclic and separating vector. Hence $\varphi_{01}$ restricted to $\mathcal{O}$ is unitarily implementable ([8], Chap. I, § 4, subsection 3, Prop. 1).

We now would like to apply Theorem 21. But it is first necessary to know the center of the global algebra $\varphi_{1}(A)$. We will separate the investigation of this question from the proof of Theorem 23. The answer to this question is

Theorem 26. Let $L$ be any linear positive map of $\mathfrak{A}(\mathscr{M})$ into $\mathfrak{C}(\mathscr{M})$ fulfilling the conditions 1 )-5) of Theorem 23. Let $\Psi$ be a separating vector for $\mathfrak{S}(\mathscr{M})$ and $\omega(A)=(\Psi, L(A) \Psi)$. If $\varphi(A)$ denotes the canonical representation induced by $\omega(A)$, then the center of the v. Neumann algebra generated by $\varphi(A)$ is the image of $\mathfrak{S}(\mathscr{M})$ under the isomorphism $\varphi$ :

$$
\mathfrak{S}(\mathfrak{R}\{\varphi(A)\})=\varphi(\mathfrak{S}(\mathscr{M})) .
$$

Before we prove this theorem we want to show that Theorem 23 follows from it. But this is clear because $\varphi_{01}$ now fulfils all conditions of Theorem 21. Hence we have

$$
\left(\Psi, L_{0}(A) \Psi\right)=\left(\Psi, L_{1}(A) \Psi\right)
$$

for every vector $\Psi$ which is separating for the center. This then implies

which was to be shown.

$$
L_{0}(A)=L_{1}(A)
$$

Proof of Corollary 24 and 25. Theorem 23 implies that the definition of $L(A)$ in Theorem 1 is independent of the special choice of the spacelike vector $a$ and the sequence $\left\{\lambda_{n}\right\}$. This, together with the invariance properties of $L(A)$, gives Corollary 23. Corollary 24 is then an immediate consequence of Corollary 23. 
Proof of Theorem 26. The positive linear map $L$ is continuous in the strong operator topology and normal for every bounded open region. This means we are actually not dealing with $\mathfrak{A}(\mathscr{M})$ but with a bigger algebra $\mathfrak{H}_{1}(\mathscr{M})$, which is the smallest norm-closed algebra containing all $\mathfrak{R}(\mathcal{O})$.

The proof of Theorem 26 is based on a result of B. MisRA [15], which we need in a slightly generalized form.

Lemma 27. The algebra $\mathfrak{A}_{1}$ is the direct integral of simple algebras.

Let us first assume Lemma 27 and show that Theorem 26 holds. Since $\varphi(A)$ extends from $\mathfrak{A}(\mathscr{M})$ to $\mathfrak{A}_{1}(\mathscr{M})$ and $\varphi$ is faithful for $\mathfrak{A}_{1}(\mathscr{M})$, we have that the two-sided ideals of $\mathscr{H}_{1}(\mathscr{M})$ and $\varphi\left(\mathscr{H}_{1}(\mathscr{M})\right)$ are in oneto-one correspondence. Lemma 27 tells us that we have a one-to-one correspondence between the two-sided ideals of $\mathfrak{A}(\mathscr{M})$ and $\mathfrak{S}(\mathscr{M})$ resp.

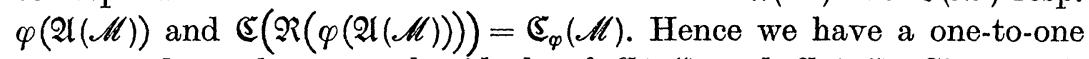
correspondence between the ideals of $\mathfrak{\mathfrak { S }}(\mathscr{M})$ and $\mathfrak{\mathfrak { V }}_{\varphi}(\mathscr{M})$. Since $\varphi$ is defined on $\mathfrak{S}(\mathscr{M})$ we have

$$
\varphi(\mathfrak{C}(\mathscr{M}))=\mathfrak{夭}_{\varphi}(\mathscr{M}) .
$$

Proof of Lemma 2\%. B. Misra proved a special form of this lemma, namely he assumed, first, that the ring $\Re(\mathscr{M})$ is a factor, and second, that for all $\mathcal{O}$ there exists an $\mathcal{O}_{1} \supset \mathcal{O}$ such that $\mathfrak{R}\left(\mathcal{O}_{1}\right)$ is a factor. These arguments are easily generalized to the case in which $R(\mathscr{M})$ contains a center and $\mathfrak{E}\left(\mathcal{O}_{1}\right)$ coincides with $\mathfrak{E}(\mathscr{M})$. But it is actually not necessary to make the assumption $\mathfrak{S}\left(\mathcal{O}_{1}\right)=\mathfrak{E}(\mathscr{M})$, since we can use our Theorem 8 and the argumentation given in the proof of Theorem 18.

\section{References}

[1] BorChers, H. J., R. HAAG, and B. Schroer: Nuovo Cimento 29, 148 (1963).

[2] ARAKI, H.: Lecture Notes (Zürich, 1962). - HAAG, R., and D. KASTLER: J. Math. Phys. 5, 848 (1964).

[3] SEqaL, I. E.: Illinois J. Math. 6, 500 (1962).

[4] ARAKI, H.: On the Algebra of all local Observables, preprint, see proposition 1.

[5] REEH, H., and S. Schuteder: Nuovo Cimento 22, 1051 (1961)

[6] NatMark, M. A.: Normed Rings. Groningen: P. Noordhoff.

[7] BorChers, H. J.: Nuovo Cimento 33, 1600 (1964).

[8] DixmזrR, J.: Les Algèbres d'Opérateurs dans l'Espace Hilbertien. Paris: Gauthier-Villars 1957.

[9] Bremermann, H. J., R. OeHue, and J. G. Taylor: Phys. Rev. 109, 2178 (1958).

[10] Borchers, H. J., and W. Zimmermans: Nuovo Cimento 31, 1047 (1964).

[11] Schwartz, L.: Théorie des Distributions. Paris: Hermann \& Cie. 1950.

[12] Borchers, H. J.: Nuovo Cimento 19, 787 (1961).

[13] Neumann, J. von: Collected Works. Vol. III, p. 64-67. Oxford: Pergamon Press 1961.

[14] Borchers, H. J.: Commun. math. Phys. 1, 8 (1965).

[15] MrsRa, B.: On the algebra of quasi-local operators of quantum-field theory, preprint. 